\title{
Dynamic characteristics of vibrated droplets on the one-tier and two-tier surface
}

\author{
Gang Wang ${ }^{1, a}$, Zhi-hai Jia ${ }^{2, b^{*}}$ and Zhen Teng ${ }^{3, c}$ \\ ${ }^{1,2,3}$ School of Energy and Power Engineering, University of Shanghai for Science and Technology, \\ Shanghai, 200093, China
}

awanggang_usst@163.com, bzhhjia@usst.edu.cn, ctengzhen456@163.com

Keywords: surface; droplet; vibration;

Abstract. Dynamic characteristics of droplets on the one-tier surface, which was a micropillared hydrophobic surface fabricated by lithography and two-tier surface, which was a micro-nano hydrophobic surface replaced by dry lotus leaf were studied under vertically vibration. Our experiments shown that the two-tier surface led to a decrease of the critical amplitude (which was required to make the droplets separated from the surface) than the one-tier surface at each given frequency. Meanwhile, droplets on the two-tier surface need lower vibration energy to separate from the surface. But the vibration morphology and mode of the droplet on the two-tier surface are rather more complicated. The physical mechanism of droplet separation by vibration was presented and then analyzing the experimental phenomenon in combination with surface mechanics and kinetic energy. We demonstrate that the length of the triple contact line is an important parameter in the form of droplet vibration mode and morphology.

\section{Introduction}

Hydrophobic or superhydrophobic surfaces are widely used in many fields to prevent corrosion [1] and reduce drag in pipelines because their hydrophobic and self-cleaning properties [2]. These surfaces have attracted great interests for many potential applications including biotechnology [3], anti-icing and thermal systems [4].

Research shows that two kinds of typical droplet wetting state are existed on rough surfaces, Wenzel state and Cassie state. Moreover, the transition between Cassie and Wenzel state has been realized by external stimuli such as pressure [5], vibration [6,7,8,9], electric voltage [10], evaporation [11], or heating [12]. Noblin et al. [13] studied the morphology change of droplets in vibration, and found that droplets on hydrophobic surface exist two types of deformation under the action of vibration, and the two types of deformation also each contains two different patterns. Chen et al. [14] found that the two-tier roughness is essential for preventing catastrophic, irreversible wetting of superhydrophobic surfaces. Bormashenko [15] argued that the droplet wetting transition took place until the constant force per unit length of the triple contact line above a certain threshold under the vibration. Chen [16] researched the wetting state transition of condensation droplets on a lotus leaf under the action of vibration and considered that when the kinetic energy provided by the speaker was twice more than the adhesion energy. The condensate droplets would achieve the Wenzel-Cassie state. Extrand et al. [17] modeled the contact angles and sliding angles exhibited by the lotus leaf, accounting for the influence of curvature and pinning. Bhushan [18] reported that when the droplet inertia force $F_{1}$ provided by vibration was greater than the adhesive force between the droplet and surface $F_{A}$, the droplet would vertically separate from the surface and achieved the wetting transition. Bormashenko [19] suggested that the interplay of capillary and disjointing pressures governed the equilibrium of the liquid in the nano-micrometrically scaled pores constituting the relief of the surface.

However, few studies have been reported on the droplet dynamic characteristics of the one-tier and two-tier hydrophobic surface under vibration, and the droplet vibration morphology and mode are not clear. Therefore, in this paper, droplet dynamic characteristics and deformation mode by applying vibration to the one-tier and two-tier surface were researched, laid the foundation for a better realization of droplet wetting transition on micro or micro-nano hydrophobic surfaces by vibration. 


\section{Experimental section}

One-tier hydrophobic surfaces were fabricated using PDMS material by soft lithography. The PDMS stamps were prepared in a replication molding process using a 6 inch silicon wafer covered with a microstructured photoresist layer as a master. For the preparation of the master, a layer of photoresist (SU-8, formulation 25, a new negative near-UV thick photoresist) was spin-coated on a hydrophilic silicon wafer. Subsequently, the photoresist was patterned with the desired features, using a printed foil mask. The polymer was allowed to cure for one hour at $90^{\circ} \mathrm{C}$ and to be cooled down to room temperature before being removed from the master. The convex structure on surface was fabricated using micro processing etching method as shown in Fig 1(a). Two-tier superhydrophobic surfaces were fabricated using dry lotus leaves which contained lotus leaf mastoid (micro-sized) and lotus leaf hairs (nano-structure) as shown in Fig 1(b).
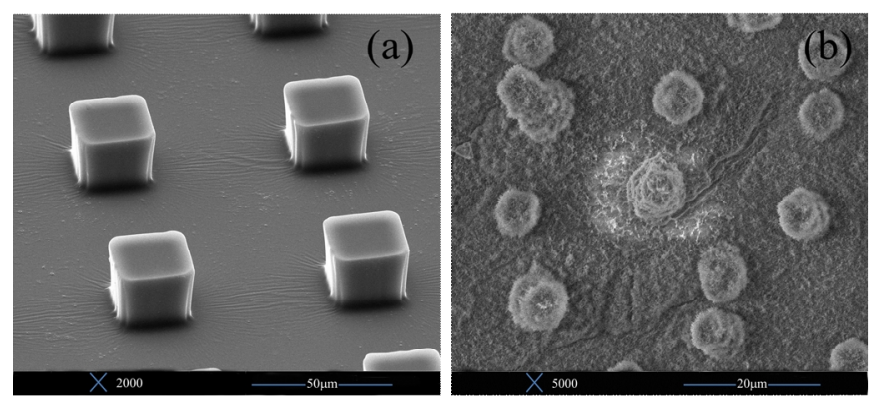

Fig. 1. SEM images of (a) one-tier (PDMS) and (b) two-tier (lotus leaf) roughness surface with the QUANTA FEG 450 field emission environmental scanning electron microscope (minimum resolution of $1 \mathrm{~nm}$ under low vacuum)

For samples were prepared, the experimental setup is shown in Fig. 2. A droplet which was introduced by deionized water was rested on samples and the droplet was vertically vibrated with a loudspeaker. The vibration signal was produced by a function generator and then connected to an amplifier. The maximal amplitude was $4 \mathrm{~mm}$ and the vibrational frequency was varied from 5 to $200 \mathrm{~Hz}$. To measure the droplet state and surface vibration amplitude, a CCD system (OLYMPUS i-SPEED3) was used at a rate of 2000 frames/s.

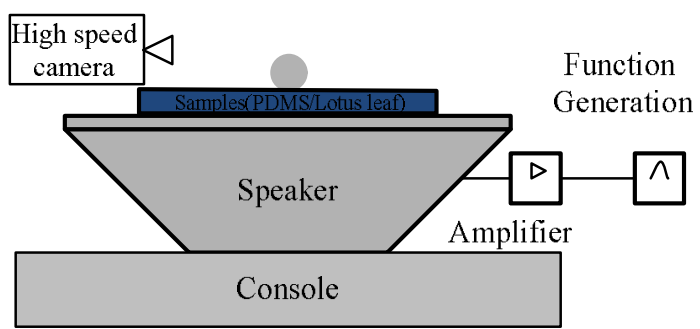

Fig. 2. Schematic of experimental setup

\section{Results and discussion}

To observe how the one-tier and two-tier structure affect the dynamic characteristics, experiments with a droplet on different samples were performed. The experiment obtained minimum energy values that could induce the droplet separated from the different surfaces as a function of frequency is shown in Fig. 3. In experiments, under a given frequency, the amplitude threshold was experimentally determined by gradually increasing the amplitude until the droplet separated from the surface. Repeating the experiments and removing the error bars and then calculating an average for each frequency. Fig. 3 shows that the droplets on the two-tier surface which volume is $4 \mu \mathrm{L}, 6 \mu \mathrm{L}, 8 \mu \mathrm{L}$, are easier to separate from the surface than the ones on the one-tier surface. The explanation for this phenomenon was associated with adhesion energy which was closely related to the solid-liquid contact 
area. When the vibration amplitude was small, the energy imparted by the vibrating speaker couldn't overcome the adhesion energy between the liquid and solid surface. Under a fixed frequency, increasing the amplitude, when the vibrating energy was greater than the adhesion energy, the droplet would be detached from the surface. However, as a result of the existence of lotus leaf hairs, the solid-liquid contact area of the droplet on the two-tier surface was smaller than the one-tier surface, so the droplet on the two-tier surface needed smaller vibrating energy to separate from the surface.
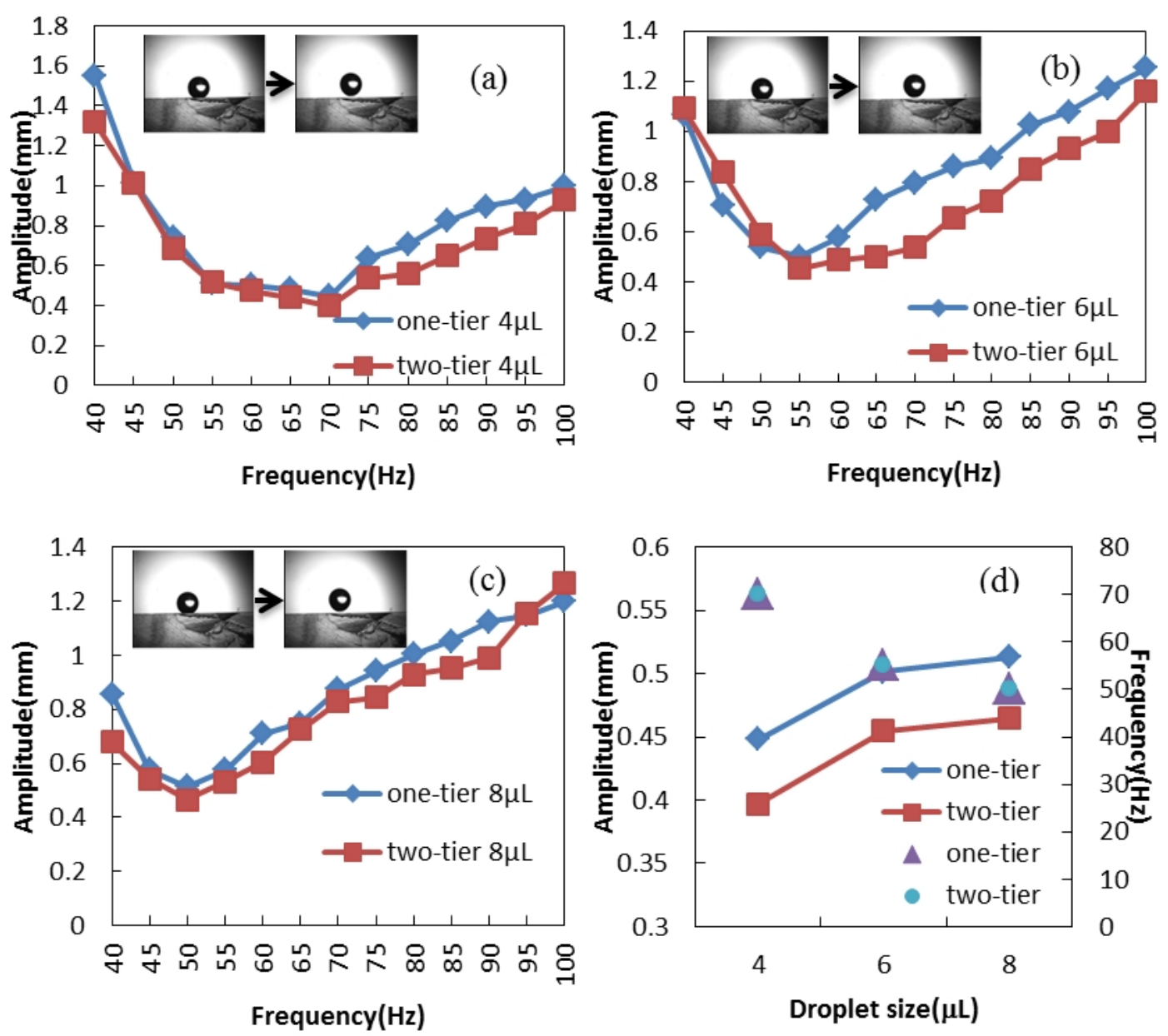

Fig. 3. Threshold amplitude of the dewetting caused by vibration, droplet which volume was 4 to 8 $\mu \mathrm{L}$ on the one-tier and two-tier surface under different frequency were shown in (a), (b), (c)

Also in the Fig. 3, each curve existed a minimum amplitude, for a $4 \mu \mathrm{L}$ droplet, at specific frequency ranges around $70 \mathrm{~Hz}$, a resonance mode was indicated where the droplet easily bounced off from the surface with a slight external vibration. A relatively large vibration apptitude was needed to separate droplets from the surface at other frequencies.

For droplets on the vibration surface, the inherent frequency $f_{n}$ was [18]:

$$
f_{n}=\sqrt{j(j+1)(j+2) \gamma /(3 \pi \rho V)}
$$

Where $\rho$ was droplet density; $\gamma$ was liquid surface tension; $j$ was droplet vibration mode number; and $V$ was the volume of the droplet in this work.

In this paper, there were two regimes of axi-symmetric oscillations for the droplet. One was the contact line fixed and the other was the contact line oscillates. When the contact line was depinned, two scenarios existed, $k=1$ or $k=2$ [13]: 


$$
k=j-\frac{1}{2}
$$

From the experimental results shown in Fig. 4 we could find that the triple contact line between droplet and hydrophobic surface continuous changed. It belonged to the $k=1$ mode and found they have two nodes by analyzing the images in Fig. 4.

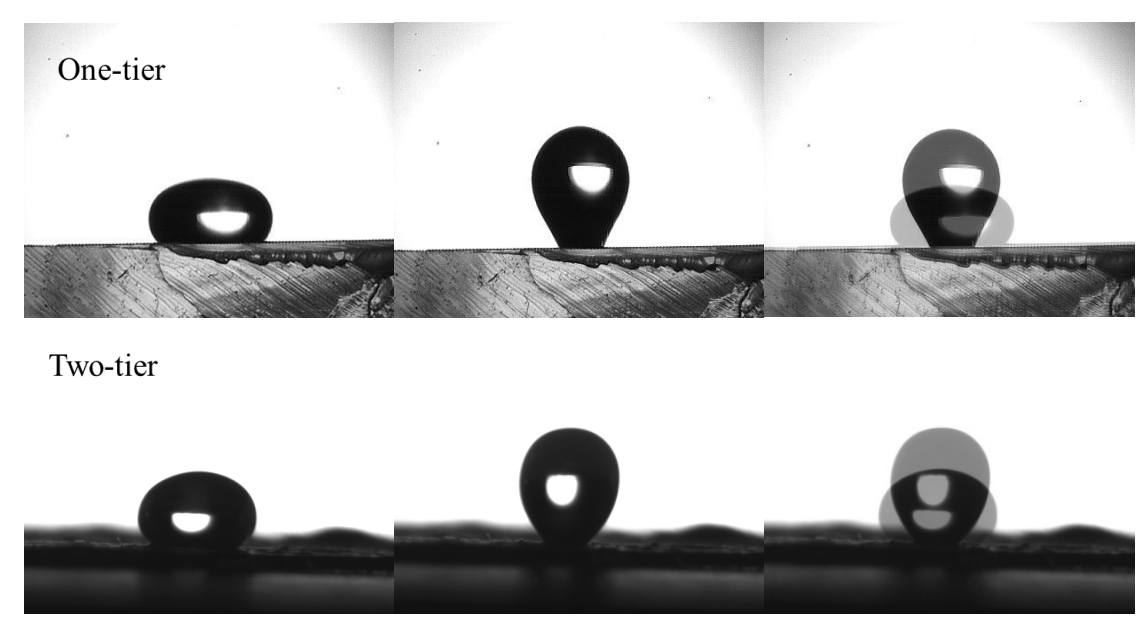

Fig. 4. Droplet vibration Mode k=1 (two nodes)

Then $j=k+1 / 2=3 / 2$, the contact liquid was deionized water, which had a density of $\rho$ was $998 \mathrm{~kg} / \mathrm{m}^{3}$ and a surface tension of $\gamma$ was $72.8 \mathrm{mN} / \mathrm{m}$, by using the Eq. 1 we got the resonant frequency was $f_{n}$ was $71.3 \mathrm{~Hz}$ when the droplet volume was $4 \mu \mathrm{L}, f_{n}$ was $57.9 \mathrm{~Hz}$ when the droplet volume was $6 \mu \mathrm{L}$, and $f_{n}$ was $50.4 \mathrm{~Hz}$ when the droplet volume was $8 \mu \mathrm{L}$, which had no significant difference with the measured value ( $f$ was $70 \mathrm{~Hz}$ for $4 \mu \mathrm{L}$ droplet; $f$ was $55 \mathrm{~Hz}$ for $6 \mu \mathrm{L}$ droplet; and $f$ was $50 \mathrm{~Hz}$ for $8 \mu \mathrm{L}$ droplet as shown in Fig. 3). When the surface vibration frequency was equal to the natural frequency of droplet, that was to say, in the resonance state, the minimum kinetic vibration energy for droplets separation was needed. As the Fig. 3(d) shows that the minimum detaching amplitude of droplet gradually increasing with the increase of droplet size, and the droplet on the two-tier surface minimum amplitude was $0.05 \mathrm{~mm}$ smaller than the one-tier surface.

For a sessile droplet on a hydrophobic surface, the droplet would be separated from the surface only when the vibration energy $E_{k}$ was larger than the surface adhesion energy $E_{a}$ between the droplet and the surface.

$$
E_{a}<E_{k}
$$

Therefore, for a vibrated surface which the vibration frequency was $f$ and the amplitude was $A$, the kinetic energy imparted to the droplet $E_{k o}$ was [16]:

$$
E_{k o} \propto \frac{1}{2} \rho V(2 \pi f A)^{2}
$$

Where $\rho$ and $V$ was the droplet density and volume, $f$ was vibration frequency, Hz; and $A$ was vibration amplitude, mm. The work of adhesion between liquid and solid phase was:

$$
E_{a}=\left(\gamma_{L A}+\gamma_{S A}-\gamma_{S L}\right) \Delta A
$$

Where $\gamma_{L A}, \gamma_{S A}, \gamma_{S L}$ were the surface tensions of the liquid-air, solid-air, and solid-liquid interface, $\Delta A$ was the wetting area. 
As a result of the existence of the secondary structure, the wetting area of the droplet on the two-tier surface was smaller than the one-tier surface, so the corresponding adhesion energy was smaller, when the same frequency and droplet were given, the droplet on the two-tier surface needed lower vibration amplitude to overcome the adhesion energy, and this agreed reasonably well with experimental observations.

The experiments reveal that the droplet vibration form on the two-tier surface diversified when the droplet volume was big (nearly $10 \mu \mathrm{L}$ or larger), not only mode $k=1$ like droplet on the one-tier surface, related images as shown in Fig. 5.

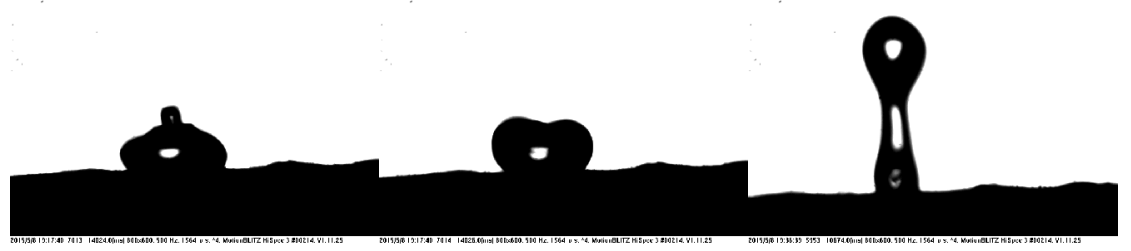

Fig. 5 Droplet patterns on two-tier surface

Due to the lower adhesion energy of droplet on the two-tier surface, when the vibration was given and the volume of droplet was big, with the wetting area $\Delta A$ gradually decreased, the interfacial energies of droplet would change sharply, so the droplet should have a diversity of morphology.

\section{Conclusions}

We anticipate that the micro-nano structure could influence the dewetting transition by vibration, and the droplet on the two-tier surface needs a lower vibration energy. In the process of vibration, the droplet which size is big tends to have a variety vibration pattern, and its vibration mode is no longer onefold mode, on the contrary, the vibration mode is stable no matter on the one-tier surface or two-tier surface when the droplet size is small. Our theoretical analysis agrees well with experimental observations, accounting for the influence of the one-tier or two-tier surface on droplet dynamic characteristics.

\section{Acknowledgements}

The authors wish to acknowledge the funding supports from the National Natural Science foundation of China (Grant No. 51176123), Specialized Research Fund for the Doctoral Program of Higher Education (Grant No.20103120120006), and Shanghai Natural Science (Grant No.11ZR1424800).

\section{Corresponding Author}

*E-mail: zhhjia@usst.edu.cn.

\section{References}

[1] M. Reyssat, D. Richard, C. Clanet, D. Quéré, Faraday discussions. 146 (2010) 19-33.

[2] N.L. Tarwal, P.S. Patil, Applied surface science. 256 (2010) 7451-7456.

[3] C.H. Choi, C.J. Kim, Langmuir. 25 (2009) 7561-7567.

[4] A.R. Betz, J. Jenkins, D. Attinger, International Journal of Heat and Mass Transfer. 57 (2013) 733-741.

[5] P. Forsberg, F. Nikolajeff, M. Karlsson, Soft Matter. 7 (2011) 104-109. 
[6] E. Bormashenko, R. Pogreb, G. Whyman, Y. Bormashenko, and M. Erlich, Applied physics letters. 90 (2007) 201917.

[7] H.Y. Kim, Physics of Fluids. 16 (2004) 474-477.

[8] W. Lei, Z.H. Jia, J.C. He, T.M. Cai, Applied Thermal Engineering. 62 (2014) 507-512.

[9] W. Lei, Z.H. Jia, J.C. He, T.M. Cai, and G. Wang, Applied Physics Letters. 104 (2014) 181601.

[10] G. Manukyan, J.M. Oh, D. van den Ende, R. G. H. Lammertink, and F. Mugele, 106 (2011) 014501.

[11] C. Luo, M. Xiang, X. Liu, H. Wang, Microfluidics and Nanofluidics. 10 (2011) 831-842.

[12] G. Liu, L. Fu, A.V. Rode, V.S.J. Craig, Langmuir. 27 (2011) 2595-2600.

[13] X. Noblin, A. Buguin, F.B. Wyart, Soft Matter and Biological Physics. 14 (2004) 395-404.

[14] J.B. Boreyko, C.H. Baker, C.R. Poley, C.H. Chen, Langmuir. 27 (2011) 7502-7509.

[15] E. Bormashenko, A. Musin, G. Whyman, M. Zinigrad, Langmuir. 28 (2012) 3460-3464.

[16] J.B. Boreyko, C.H. Chen, Physical review letters. 103 (2009) 174502.

[17] C.W. Extrand, S.I. Moon, Langmuir. 30 (2014) 8791-8797.

[18] Y.C. Jung, B. Bhushan, Langmuir. 25 (2009) 9208-9218.

[19] E. Bormashenko, V. Starov, Colloid and Polymer Science. 291 (2013) 343-346. 